\title{
MÉTODOS PARA INSERÇÃO DA SUSTENTABILIDADE NO DESENVOLVIMENTO DE SISTEMAS: REVISÃO SISTEMÁTICA DA LITERATURA
}

Iana Castelo Branco Giesbrecht [ iana.giesbrecht@gmail.com ] - Programa de PósGraduação em Sistemas Mecatrônicos - Universidade de Brasília

Andréa Cristina dos Santos [ andreasantos@unb.br] - Programa de Pós-Graduação em Sistemas Mecatrônicos - Universidade de Brasília

\section{RESUMO}

O objetivo deste trabalho é investigar o projeto para sustentabilidade, identificando os métodos e técnicas utilizados nos diversos modelos de desenvolvimento de produto/sistema para inserção da sustentabilidade, equilibrando os aspectos econômico, ambiental e social. Partindo da premissa que os trabalhos acadêmicos de projeto para sustentabilidade oferecem pouca ênfase a uma visão de sistemas, envolvendo os três aspectos de forma equilibrada, ou, seja, os aspectos sociais para geração de renda (econômico) são menos enfatizados quando comparados aos aspectos ambientais no contexto de tecnologias apropriadas. A revisão consistiu de três etapas principais. A primeira atividade da revisão sistemática é a separação dos eixos da pesquisa em Engenharia de Sistemas, Sustentabilidade e Projeto de produto. Em seguida, o processo segue com outras três principais etapas: a primeira de seleção dos artigos, a segunda de verificação dos artigos e a terceira de leitura. A seleção dos artigos prevê a definição das palavras chave e do banco de dados onde foi feita a busca, para tal foi utilizada a base Web of Science com restrição no período de 2006 e 2016. As palavras chave definidas, foram utilizadas com status Topic para que fossem pesquisadas ao mesmo tempo no título, palavras chave e resumo dos artigos. A busca resultou na quantidade de 196 artigos brutos. Esses foram então analisados com intuito de focar naqueles que contribuíram com a pesquisa. Dessa forma, 31 artigos foram selecionados e tiveram seu conteúdo analisado segundo os critérios: a relação da ênfase do trabalho com os três eixos da sustentabilidade; a fase de aplicação do trabalho no processo de desenvolvimento de produto; o setor da economia que o trabalho foi aplicado e as ferramentas utilizadas. A leitura destes artigos resultou em diferentes análises que suportam o desenvolvimento da pesquisa. A revista que teve um maior número de artigos analisados foi a Journal of Cleaner Production com 14 trabalhos. O ano de 2015 foi concentrador das publicações com 9 artigos frente a 4 de outros anos. Com isso, foi possível mapear de que maneira a comunidade acadêmica tem colocado em prática $o$ desenvolvimento de sistemas considerando a sustentabilidade.

Palavras chave: Design for Sustainability, DFS, Engenharia de Sistemas, Projeto de Produto

Área: Melhoria do Processo de desenvolvimento de serviços 


\section{INTRODUÇÃO}

A abordagem sustentável, tem ganhado cada vez mais importância, este enfoque baseado na harmonização dos objetivos sociais, ecológicos e econômicos, antes de ser chamado de sustentável, foi denominado por Ignacy Sachs em seu trabalho por ecodesenvolvimento (SACHS, 1997).

Segundo Diniz (2008), quando este conceito foi proposto o autor falava de novos tipos de desenvolvimento e de novos modos de produção e estilos de vida, baseando-se nas condições dos ecossistemas e da gestão dos recursos, adaptados a cada região ou diversidade étnica ou ainda a capacidade de uma gestão participativa dos recursos por parte das populações. Do mesmo modo, o final da década de 1990 viu o conceito do triple bottom line (TBL) decolar. Elkington (2004), em seu livro expõe que o termo, criado em 1994, teve origem na observação de que as dimensões sociais e econômicas da agenda proposta pelo Relatório Brundtland, de 1987 (WCED, 1987) tinham de ser abordadas de uma forma mais integrada visando um verdadeiro progresso ambiental. Desse modo, o TBL se tornou a representação mais difundida do conceito de desenvolvimento sustentável ao referir-se à integração das dimensões ambiental, social e econômica.

Para Jayswal (2011) um projeto sustentável deve basear-se na concepção e realização de uma determinada atividade econômica considerando suas consequências nas dimensões ambiental e social. Implementar a filosofia de sustentabilidade no início do processo de projeto de um produto ou sistema requer muito menos esforço e custo do que para adaptar o processo após o seu lançamento. A sustentabilidade é um assunto complexo, porque geralmente inclui vários subsistemas, muitos objetos e variáveis que mudam dinamicamente, os quais acontecem de serem difíceis de medir e possuem valores que são difíceis de calibrar (RANKY, 2015

A concepção de um produto ou sistema é um processo sistemático de identificação, exploração e desenvolvimento de oportunidades de valor (LEE; PAREDIS, 2014). Mais especificamente, o Projeto para Sustentabilidade diz respeito a balancear aspectos econômicos, ambientais e sociais na criação de produtos e serviços. Além de também pensar em todo ciclo de vida do produto e seus principais benefícios (TISCHNER; CHARTER, 2001).

Nesse sentido, o objetivo deste trabalho é investigar o projeto para sustentabilidade, identificando os métodos e técnicas utilizados nos diversos modelos de desenvolvimento de produto/sistema para inserção da sustentabilidade, equilibrando os aspectos econômico, ambiental e social. Partindo da premissa que os trabalhos acadêmicos de projeto para sustentabilidade oferecem pouca ênfase a uma visão sistêmica, envolvendo os três aspectos de forma equilibrada, no qual os aspectos sociais para geração de renda (econômico) são menos enfatizados quando comparados aos aspectos ambientais no contexto de tecnologias apropridas.

Para tanto, foi desenvolvida uma revisão bibliográfica que apresenta os resultados deste tema de 2006 até 2016. A estrutura do artigo inicia com a descrição do método que foi utilizado para a pesquisa. Em seguida, são apresentadas as classificações criadas para a análise dos resultados subsequentes. Por fim, são comentadas as considerações finais obtidas no trabalho.

\section{MÉTODO DA PESQUISA}

O artigo é de natureza exploratória porque reúne dados e informações a respeito dos temas selecionados os quais foram analisados segundo abordagem quantitativa a fim de caracterizar 
sua natureza teórica conceitual. Foi utilizado como método de levantamento bibliográfico a revisão sistemática. Em seguida à revisão é apresentada uma análise estruturada dos temas explorados.

A revisão está dividida em atividades sequenciais conforme apresentado na Figura 1. E resultou primeiramente em um Banco de Artigos bruto, este banco foi revisado para finalizar como Banco de Artigos da pesquisa e assim subsidiar a análise estrutura posterior.

A primeira atividade da revisão foi a definição dos eixos da pesquisa: Engenharia de Sistemas, Sustentabilidade e Projeto de Produto, ilustrados na Figura 2.

No contexto da Engenharia de Sistemas, buscou-se compreender de que maneira a academia estava inserindo os sistemas sócio técnicos como resultado do processo de desenvolvimento de sistemas. No caso da sustentabilidade era importante explorar se o conceito de Design for “ $X$ ”, mais especificamente no caso do Design for Sustainability, e se o mesmo considerava as tecnologias apropriadas. Ao final, o tema de Projeto de Produto foi estudado para caracterizar seus métodos de apoio ao projeto e entender cada uma das etapas do processo.

Em seguida, foram definidas as palavras chave e pesquisadas com status TOPIC para que fossem buscadas ao mesmo tempo no título, palavras chave e resumo dos artigos. A Tabela 1 reúne as palavras chaves da revisão. O Banco de Dados que foi utilizado foi o Web of Science com restrição no período de 2006 a 2016. Tendo sido obtida a lista com os artigos como resultado da pesquisa, eles foram acessados através da base da CAPES (Coordenação de Aperfeiçoamento de Pessoal de Nível Superior). Essas duas bases foram selecionadas, primeiro pela abrangência na pesquisa e segundo pelo acesso livre aos textos completos.

Figura 1. Processo de revisão sistemática da pesquisa. Elaborado Autores

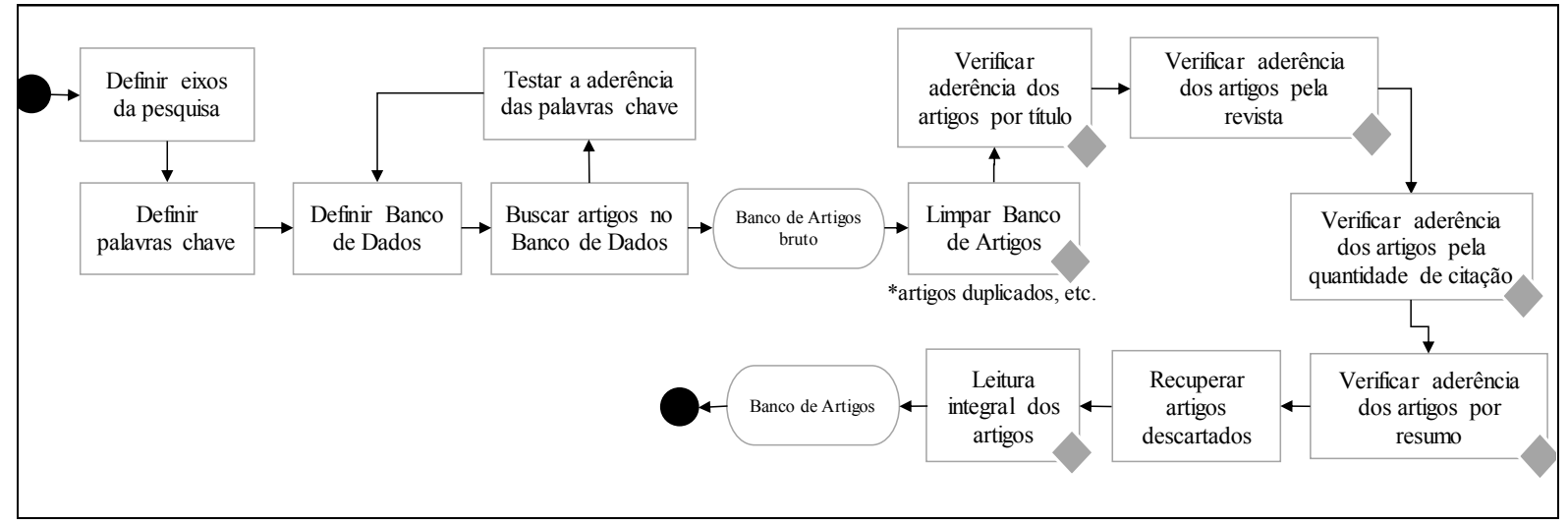

Figura 2. Eixos da pesquisa. Elaborado Autores

\begin{tabular}{|c|c|c|}
\hline \multicolumn{3}{|l|}{ Eixos da Pesquisa: } \\
\hline Engenharia de Sistemas & Sustentabilidade & Projeto de Produto \\
\hline $\begin{array}{l}\text { Processo de desenvolvimento de Sistemas } \\
\text { Sistemas Sócio Técnicos }\end{array}$ & $\begin{array}{l}\text { Tecnologias apropriadas } \\
\text { DFX }\end{array}$ & $\begin{array}{c}\text { Métodos de Desenvolvimento } \\
\text { Processo de Desenvolvimento de Produto } \\
\text { Concepção }\end{array}$ \\
\hline
\end{tabular}


Tabela 1: Palavras chave e resultado da busca na base.

\begin{tabular}{|c|c|c|c|c|c|}
\hline & Palavra Chave & $\begin{array}{l}\text { Results } \\
\text { found }\end{array}$ & $\begin{array}{c}\text { Sum of } \\
\text { the } \\
\text { Times } \\
\text { Cited }\end{array}$ & $\begin{array}{l}\text { Average } \\
\text { Citations } \\
\text { per Item }\end{array}$ & $\begin{array}{c}\text { h- } \\
\text { index }\end{array}$ \\
\hline PC1: & "Systems Engineering" + "Sociotechnical system" & 5 & 43 & 8.6 & 1 \\
\hline PC2: & $\begin{array}{l}\text { "Systems Engineering" + "Methods" + } \\
\text { "Sustainability" }\end{array}$ & 11 & 132 & 12 & 7 \\
\hline PC3: & "Product Design" + "Tools" + "Sustainability" & 25 & 489 & 19.56 & 9 \\
\hline PC4: & "Engineering Design"+ "Best Practices" & 16 & 109 & 6.81 & 4 \\
\hline PC5: & "Design for sustainability" & 60 & 478 & 7.97 & 12 \\
\hline PC6: & "Sociotechnical system" & 68 & 865 & 12.72 & 15 \\
\hline PC7: & "Design for Environment" + "Design for Cost" & 1 & 0 & 0 & 0 \\
\hline \multirow[t]{2}{*}{ PC8: } & "DFX" + "Sustainability" & 10 & 4 & 0.4 & 1 \\
\hline & SOMA: & 196 & 2120 & 68.06 & 49 \\
\hline
\end{tabular}

Nesta busca à base foram encontrados 196 artigos, Tabela 1, os quais compuseram a Base de Artigos bruta. Para a análise e classificação dos artigos foram utilizados os seguintes critérios, nesta ordem: título, aderência da revista ao tema, quantidade de citação, aderência do resumo ao tema e, por fim, foi feita a leitura integral do trabalho. O resultado dessa classificação é apresentado no tópico a seguir.

\section{CLASSIFICAÇÃO DOS ARTIGOS ANALISADOS}

Os 196 artigos selecionados foram verificados quanto à duplicidade, à aderência do título, ao escopo das revistas e à quantidade de citações. O título foi analisado subjetivamente um a um para que fossem selecionados apenas aqueles que têm relação com os eixos da pesquisa.

A análise das revistas e da quantidade das citações foi realizada apenas nos artigos que permaneceram após a análise dos títulos. Então, a lista das revistas foi analisada para retirar aquelas que não correspondiam aos eixos da pesquisa. Permaneceram os trabalhos que tinham número de citações acima de 3 .

Com isso, foram excluídos 103 artigos com títulos sem relação com a pesquisa e 16 que a revista não faziam parte do escopo do estudo. Neste momento a quantidade de citação foi utilizada como critério de desempate. Desses permaneceram 77 artigos para download integral, porém, 22 estavam indisponíveis por falta de acesso à base. A Tabela 2 apresenta 0 resultado.

Tabela 2: Resultado das etapas de classificação dos artigos.

\begin{tabular}{|l|l|}
\hline Artigos retirados por TÍTULO & 103 \\
\hline Artigos retirados por REVISTA, CITAÇÃO & 16 \\
\hline INDISPONIBILIDADE do Arquivo & 22 \\
\hline Artigos retirados por RESUMO & 24 \\
\hline LISTA FINAL: & 31 \\
\hline
\end{tabular}


A leitura destes artigos resultou em diferentes análises que suportam o desenvolvimento da pesquisa. A Tabela 3 apresenta, desses 31 artigos, quais foram as revistas mais pesquisadas e seu respectivo fator de impacto. A revista que teve um maior número de artigos analisados foi a Journal of Cleaner Production com 14 trabalhos e é também a revista com maior fator de impacto 5,315. O ano de 2015 foi concentrador das publicações com 9 artigos frente a 4 de outros anos.

O trabalho de Anette (2014), mostrou que há a necessidade de explorar a relação entre a pesquisa focada no produtor e conceitos orientados ao comportamento do consumidor para melhorar a sustentabilidade do produto. Além disso, ela acredita que as dimensões econômicas e sociais da sustentabilidade podem ser impactadas pelo comportamento do usuário.

A Tabela 4 lista a classificação dos trabalhos por tipo de pesquisa, mostrando o grande número de trabalhos de Estudo de Caso seguidos de Revisão Bibliográfica e Teóricoconceitual. Os temas que foram explorados nos estudos de caso apresentados estão listados na Tabela 5 e mostram que dos eixos da pesquisa estão presentes nos mais diversos setores da economia.

Tabela 3: Resultado por revista com fator de impacto correspondente.

\begin{tabular}{|l|c|c|}
\hline \multicolumn{1}{|c|}{ Revista } & Qtd. & $\begin{array}{c}\text { Fator de } \\
\text { Impacto }\end{array}$ \\
\hline JOURNAL OF CLEANER PRODUCTION & 14 & 5.315 \\
\hline EXPERT SYSTEMS WITH APPLICATIONS & 2 & 2.879 \\
\hline COMPUTERS \& CHEMICAL ENGINEERING & 2 & 2.821 \\
\hline $\begin{array}{l}\text { 2013 5TH INTERNATIONAL CONFERENCE ON ADAPTIVE SCIENCE } \\
\text { AND TECHNOLOGY (ICAST 2013) }\end{array}$ & 1 & - \\
\hline CLEAN TECHNOLOGIES AND ENVIRONMENTAL POLICY & 1 & 2.052 \\
\hline DESIGN STUDIES & 1 & 2.652 \\
\hline HUMAN FACTORS & 1 & 1.767 \\
\hline INTERNATIONAL JOURNAL OF DESIGN & 1 & 1.209 \\
\hline INTERNATIONAL JOURNAL OF LIFE CYCLE ASSESSMENT & 1 & 4.056 \\
\hline JOURNAL OF ENGINEERING AND TECHNOLOGY MANAGEMENT & 1 & 2.19 \\
\hline JOURNAL OF ENGINEERING DESIGN & 1 & 1.949 \\
\hline JOURNAL OF INDUSTRIAL ECOLOGY & 1 & 4.094 \\
\hline CURRENT OPINION IN CHEMICAL ENGINEERING & 1 & 3.81 \\
\hline SUSTAINABILITY & 1 & 1.343 \\
\hline SYSTEMS & 1 & - \\
\hline COMPUTER-AIDED DESIGN & 1 & 2.304 \\
\hline
\end{tabular}

Foi observado que pelo tipo de pesquisa de Estudo de Caso, que os eixos da pesquisa estão presentes em diversos setores da economia. Os setores de aplicação de Estudo de Caso foram: biodiesel, automobilístico, desenvolvimento de software, biocombustível, calçados, vestuário, 
móveis, máquinas e equipamentos, eletrônicos, material esportivo, mobilidade urbana, alimentos e turismo.

Tabela 4. Classificação dos trabalhos por tipo de pesquisa.

\begin{tabular}{|l|c|}
\hline \multicolumn{1}{|c|}{ Tipo de pesquisa } & Qtd. \\
\hline Survey & 2 \\
\hline Revisão Bibliográfica & 5 \\
\hline Teórico-conceitual & 5 \\
\hline Estudo de Caso & 9 \\
\hline Revisão da Literatura & 2 \\
\hline
\end{tabular}

Em seguida, a revisão sistemática mostrou que o ano de 2015 teve a maior quantidade de artigos publicados nos temas pesquisados. Conforme mostra o Gráfico 1. Nesse sentido, se vê uma crescente na exploração dos eixos da pesquisa. Além disso, foi observado que dos 9 artigos de Estudo de Caso, 5 estavam concentrados em 2015 e 2016. Reafirmando a necessidade observada de aplicar as ferramentas na prática.

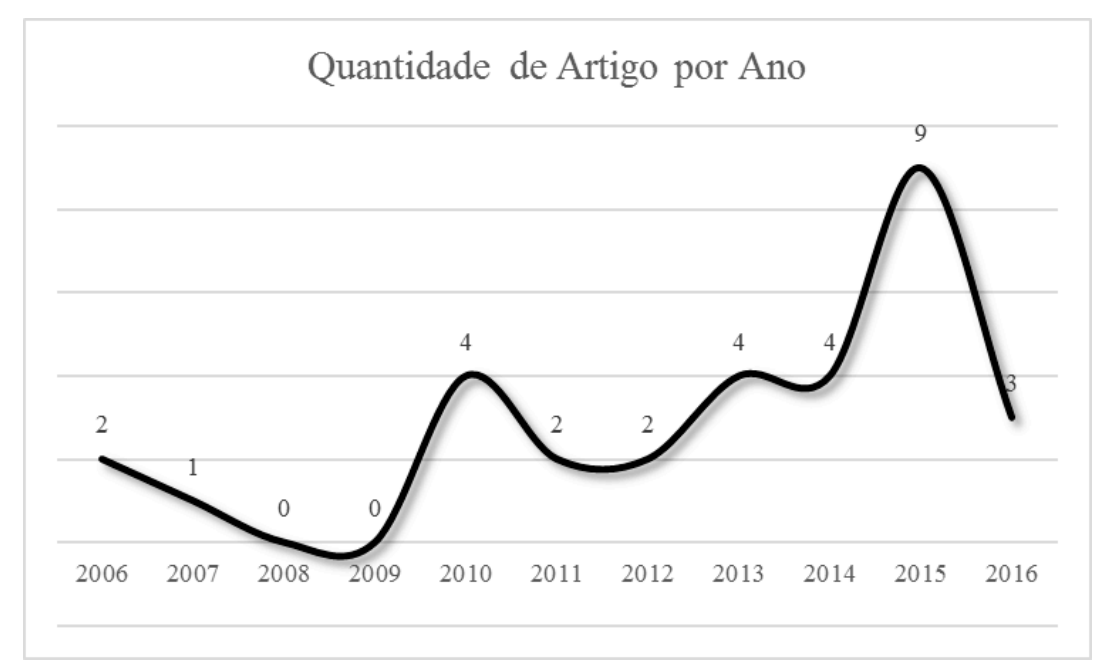

Gráfico 1. Quantidade de artigo por ano. Elaborado autores.

Os 31 artigos que foram resultado das análises tiveram seu conteúdo classificado segundo os critérios: a relação da ênfase do trabalho com os três eixos da sustentabilidade; a fase de aplicação do trabalho no processo de desenvolvimento de produto; o setor da economia que o trabalho foi aplicado e as ferramentas utilizadas.

A Tabela 5 apresenta os principais trabalhos que sugeriram ou aplicaram ferramentas especificamente com o intuito de inserir a sustentabilidade no projeto de um serviço, sistema ou produto. É possível observar a dificuldade de listar ferramentas que façam essa interação em todas as etapas do processo de desenvolvimento de produto.

A abordagem sustentável é entendida neste trabalho a partir da combinação de dois trabalhos principais: a teoria do triple bottom line com a pesquisa de Ignacy Sachs de 1997. Ambos caracterizaram a sustentabilidade a harmonização dos objetivos sociais, ecológicos e econômicos (SACHS, 1997; ELKIGTON, 2004). Cada um deles criou uma definição 
diferente para cada aspecto da sustentabilidade, mas que se complementam. No caso deste artigo, optou-se por manter a análise baseada nestes três pilares. Conforme Tabela 6.

É interessante observar primeiramente que não são todos os 31 trabalhos que estão presentes nesta análise porque alguns deles tratam a sustentabilidade de maneira ainda teórica e, como não discutem como implementá-la, também não mostram qual aspecto da sustentabilidade estão discutindo. Além disso, é importante destacar a dificuldade de se abarcar todos os três pilares: ambiental, econômico e social.

Os conceitos que foram levantados desta revisão sistemática da literatura serão concatenados no tópico a seguir.

Tabela 5. Aplicação das ferramentas nas fases do desenvolvimento de produto.

\begin{tabular}{|l|l|l|l|l|l|}
\hline \multicolumn{1}{|c|}{ Autor } & $\begin{array}{c}\text { Pré - } \\
\text { Desenvolvimento }\end{array}$ & $\begin{array}{c}\text { Projeto } \\
\text { Informacional }\end{array}$ & $\begin{array}{c}\text { Projeto } \\
\text { Conceitual }\end{array}$ & $\begin{array}{c}\text { Projeto } \\
\text { Detalhado }\end{array}$ & $\begin{array}{c}\text { Lançamento } \\
\text { do Produto }\end{array}$ \\
\hline (Luttropp e Lagerstedt, 2006) & & & & & \\
\hline (Ajukumar e Gandhi, 2013) & & & & \\
\hline (Azkarate, et al., 2011) & & & & \\
\hline (Chandrasegaran, et al., 2013) & & & & \\
\hline (Fargnoli, Minicis e Tronci, 2014) & & & & \\
\hline (Kremer, et al, 2016) & & & & \\
\hline (Luthe, Kaegi e Reger, 2013) & & & \\
\hline
\end{tabular}

Tabela 6. Aplicação dos trabalhos quanto ao tripé da sustentabilidade.

\begin{tabular}{|l|l|l|l|}
\hline \multicolumn{1}{|c|}{ Autores } & Ambiental & Econômica & Social \\
\hline (Fargnoli, Minicis e Tronci, 2014) & & & \\
\hline (Ajukumar e Gandhi, 2013) & & & \\
\hline (Anette, Brewer e Choal, 2014) & & & \\
\hline (Azkarate, et al., 2011) & & & \\
\hline (Bakshi, 2014) & & & \\
\hline (Ceschin, 2014) & & & \\
\hline (Clancy, et al., 2015) & & & \\
\hline (Romeiro, 2015) & & & \\
\hline (Gazuilusoy e Brezer, 2015) & & & \\
\hline (Jayswal, et al., 2011) & & & \\
\hline (Wood, et al., 2010) & & & \\
\hline (Luthe, Kaegi e Reger, 2013) & & & \\
\hline (Luttropp e Lagerstedt, 2006) & & \\
\hline (Kremer et al, 2016) & & \\
\hline
\end{tabular}




\section{ANÁLISE E CLASSIFICAÇÃO DOS TEMAS SOBRE O PROJETO PARA SUSTENTABILIDADE}

Como resultado da revisão sistemática apresentada, vários conceitos são explorados pelos autores listados, tais como: sistema sócio técnico sustentável, projeto para sustentabilidade, experimentos sócio técnicos, inovação social, dentre outros. Neste tópico, eles serão apresentados de maneira correlacionada.

A teoria sobre sistemas sócio técnicos sustentável destaca a importância dos aspectos sociais na organização, em vez de confiar somente em soluções técnicas para questões complexas. Isto é conseguido através do compartilhamento de conhecimento, aprendizado e inovação com um foco humano baseado em consciência compartilhada, agilidade e auto sincronização (WALKER, 2009).

Muitos métodos existentes para o projeto de produtos técnicos sustentáveis se concentram na eficiência ambiental, sem contar com uma estrutura para uma abordagem de projeto holístico e sustentável que inclua aspectos sociais, técnicos, econômicos e ambientais combinados em todo o ciclo de vida do produto e que forneça orientação sobre um produto com nível de desenvolvimento técnico (LUTHE, 2013).

Segundo Ceschin (2014), existe evolução do Design for Sustainability (DfS) nas últimas duas décadas quanto ao seu campo de ação. De fato, o foco passou de produtos únicos para serviços e sistemas produtos-serviços e, mais recentemente, para inovação social e mudanças sócio técnicas em larga escala.

Nesse sentido, a inovação social é um processo de mudança através do qual novas soluções emergem da inventividade e criatividade da variedade de atores diretamente envolvidos na resolução de problemas da vida diária: pessoas comuns, comunidades, técnicos e empreendedores de base, instituições locais e organizações da sociedade civil (CESCHIN, 2014).

\section{CONSIDERAÇÕES FINAIS}

Por meio deste estudo foi possível constatar que o tema continua em crescente discussão considerando o espaço ainda existente para exploração das ferramentas utilizadas. Entre os trabalhos destaca-se Anette (2014). A autora ressalta a necessidade na literatura de ser criado um método que melhor defina a aplicação do DFx baseado no ciclo de vida do produto, a autora coloca que há a necessidade de explorar a relação entre a pesquisa focada no produtor e conceitos orientados ao comportamento do consumidor para melhorar o equilíbrio entre os aspectos econômico, ambiental e social.

A teoria sobre sistemas sócio técnicos sustentável destaca a importância dos aspectos sociais na organização (WALKER, 2009). Muitos métodos existentes para o projeto de produtos técnicos sustentáveis se concentram na eficiência ambiental (LUTHE, 2013).

Para Kremer (2016) é especialmente oportuno preencher a lacuna existente entre diversos DfX, principalmente, entre o Design for Environment (DfE), o Design for Sustainability (DfS) e os demais. Isso porque se estará respondendo diretamente às necessidades de tomadores de decisão da indústria. Tendo em vista que eles têm uma inerente visão holística e que o DfE e o DfS podem ter interface com todos as outras abordagens do DfX, o que os diferencia dos demais (KREMER, 2016). 
Dessa maneira, o resultado final da revisão sistemática ainda aponta para a oportunidade de discussão sobre o relacionamento entre as diversas ferramentas apresentadas. De que maneira elas podem interagir no sentido de entregar um produto/ sistema sócio técnico sustentável.

\section{REFERÊNCIAS}

AJUKUMAR, V. N.; GANDHI, O. P., Evaluation of green maintenance initiatives in design and development of mechanical systems using an integrated approach, Journal of Cleaner Production, 2013.

ARNETTE, A. N.; BREWER, B. L.; CHOAL, T. Design for sustainability (DFS): the intersection of supply chain and environment, Journal of Cleaner Production, 2014.

AZKARATE, A. et al. An assessment method and design support system for designing sustainable machine tools, Journal of Engineering Design, 2011.

BAKSHI, B. R., Methods and tools for sustainable process design, Current Opinion In Chemical Engineering, 2014.

CESCHIN, F., How the Design of Socio-technical Experiments Can Enable Radical Changes for Sustainability, International Journal of Design, 2014.

CHANDRASEGARAN, S. K. et al. The evolution, challenges, and future of knowledge representation in product design systems, Computer-Aided Design, 2013.

CLANCY, G.; FROELING, M.; PETERS, G. Ecolabels as drivers of clothing design, Journal of Cleaner Production, 2015.

DE SOUZA, V. M.; BORSATO, M. Combining Stage Gate (TM) model using Set-Based concurrent engineering and sustainable end-of-life principles in a product development assessment tool, Journal of Cleaner Production, 2016.

DINIZ, J. D. (2008). Avaliação-construção de projetos de desenvolvimento local a partir da valorização de produtos florestais da Amazônia brasileira: caso da castanha-do-brasil. Brasília.

FARGNOLI, M.; DE MINICIS, M.; TRONCI, M. Design Management for Sustainability: An integrated approach for the development of sustainable products, Journal of Engineering And Technology Management, 2014.

GAZIULUSOY, A. I.; BREZET, H. Design for system innovations and transitions: a conceptual framework integrating insights from sustainablity science and theories of system innovations and transitions, Journal of Cleaner Production, 2015.

HERNANDEZ, N. V. et al. Development of an expert system to aid engineers in the selection of design for environment methods and tools, Expert Systems with Applications, 2012.

JAYSWAL, A et al. A sustainability root cause analysis methodology and its application, Computers \& Chemical Engineering, 2011.

KREMER, G. et al. Directions for instilling economic and environmental sustainability across product supply chains, Journal of Cleaner Production, 2016.

KUCUKSAYRAC, E. Design for sustainability in companies: strategies, drivers and needs of Turkey's best performing businesses, Journal of Cleaner Production, 2015. 
KUCUKSAYRAC, E.; KESKIN, D.; BREZET, H., Intermediaries and innovation support in the design for sustainability field: cases from the Netherlands, Turkey and the United Kingdom, Journal of Cleaner Production, 2015.

LIN, C. et al Developing an assessment framework for managing sustainability programs: A Analytic Network Process approach, Expert Systems with Applications, 2015.

LUTHE, T.; KAEGI, T.; REGER, J. A Systems Approach to Sustainable Technical Product Design: Combining Life Cycle Assessment and Virtual Development in the Case of Skis, Journal of Industrial Ecology, 2013.

LUTTROPP, C.; LAGERSTEDT, J. Eco Design and The Ten Golden Rules: generic advice for merging environmental aspects into product development, Journal of Cleaner Production, 2006.

MOLDAVSKA, A.; WELO, T. Development of Manufacturing Sustainability Assessment Using Systems Thinking, Sustainability, 2016.

MUKHERJEE, R.; SENGUPTA, D.; SIKDAR, S. K., Sustainability in the context of process engineering, Clean Technologies and Environmental Policy, 2015.

OOSTHUIZEN, R.; PRETORIUS, L., An Analysis Methodology for Impact of New Technology In Complex Sociotechnical Systems, 2013 5TH International Conference On Adaptive Science And Technology, 2013.

RANKY, P. G., An Integrated Architecture, Methods and Some Tools for Enhancing Sustainable Enterprises and Systems, Systems, 2015.

ROMEIRO FILHO, E. Brazilian design for sustainability: in search of a local approach, Journal of Cleaner Production, 2015.

SCHMIDT, W.P.; BUTT, F. Life cycle tools within Ford of Europe's Product Sustainability Index - Case study ford S-MAX \& Ford Galaxy, International Journal of Life Cycle Assessment, 2006.

SCOTT, K.; BAKKER, C.; QUIST, J. Designing change by living change, Design Studies, 2012.

SPANGENBERG, J. H.; FUAD-LUKE, A.; BLINCOE, K. Design for Sustainability (DfS): the interface of sustainable production and consumption, JOURNAL OF CLEANER PRODUCTION, 2010.

STRAUCH, B., Can Cultural Differences Lead to Accidents? Team Cultural Differences and Sociotechnical System Operations, Human Factors, 2010.

URBAN, R. A et al. Towards sustainability of engineered processes: Designing self-reliant networks of technological-ecological systems, Computers \& Chemical Engineering, 2010.

VEZZOLI, C. et al. New design challenges to widely implement 'Sustainable Product-Service Systems', Journal of Cleaner Production, 2015.

WAAGE, S. A. Re-considering product design: a practical road-map for integration of sustainability issues, Journal of Cleaner Production, 2007.

WALKER, G et al. Command and control: the sociotechnical perspective, Ashgate Publishing Company, 2009.

WOOD, M. D. K. L et al. Results of the first adapted design for sustainability project in a South Pacific small island developing state: Fiji, Journal of Cleaner Production, 2010. 